\title{
Comissionamento do sistema de planejamento para uso de correção de heterogeneidades: Construção de um objeto simulador de baixo custo para validação e controle de qualidade em radioterapia.
}

\author{
Treatment Planning System Commissioning for inhomogeneity correction: \\ Building a low-cost phantom for validation and quality assurance in \\ radiotherapy \\ Thallis A. Santos ${ }^{1}$, Aline Ragonezi ${ }^{1}$, Márcio Tokarski ${ }^{1}$, Bruna Biazotto ${ }^{1}$ \\ ${ }^{1}$ Universidade Estadual de Campinas, Campinas, Brasil
}

\begin{abstract}
Resumo
A implementação de correções por heterogeneidades em sistemas de planejamento de tratamentos radioterápicos melhora a exatidão do cálculo de dose em pacientes submetidos à teleterapia. Estudos mostram que planejamentos que não levam em conta a presença de heterogeneidades podem produzir uma subdosagem de até $20 \%$ para tumores de pulmão na entrega de dose. Para a validação, é necessário adquirir um dos objetos simuladores (OS) disponíveis comercialmente. Esses OS's permitem a realização dos testes recomendados pelo TRS430 (Commissioning and Quality Assurance of Computerized Planning Systems for Radiation Treatment of Cancer) e a implementação das correções de heterogeneidades. Esse trabalho comparou os OS's disponíveis comercialmente e um OS de baixo custo foi construído tanto para validação quanto à execução dos testes recomendados pelo TRS-430. Verificouse que é possível fazer um OS com um custo cerca de 20 vezes mais barato do que encontrado comercialmente.
\end{abstract}

Palavras-chave: fantoma de heterogeneidades; radioterapia; sistemas de planejamento; verificação dosimétrica.

\begin{abstract}
The implementation of inhomogeneity correction by treatment planning systems in radiotherapy improves the accuracy of dose calculation in patients treated in teletherapy machines. Studies show that planning without taking account of inhomogeneities can produce underdosages up to $20 \%$ to tumours in lung. For validation is necessary to acquire one of the commercially available phantoms. These phantoms will provide the achievement of the recommended tests of TRS430 (Commissioning and Quality Assurance of Computerized Planning Systems for Radiation Treatment of Cancer) and the implementation of inhomogeneity corrections. This work compared commercially available phantoms and makes a construction of a low-cost phantom that provides all the necessary requirements of TRS-430. It was possible to make a phantom 20 times cheaper then the commercially available.
\end{abstract}

Keywords: inhomogeneity phantom; radiotherapy; treatment planning system; dose verification.

\section{Introdução}

A implementação de correções por heterogeneidades em sistemas de planejamento de tratamentos radioterápicos melhora a exatidão do cálculo de dose em pacientes submetidos à teleterapia. Estudos mostram que planejamentos que não levam em conta a presença de heterogeneidades podem produzir uma subdosagem de até $20 \%$ para tumores de pulmão na entrega de dose[1, 2].

O número de CT ou Unidade de Hounsfield é empregado em imagens tomográficas para representar a relação entre a atenuação linear de um dado material em relação a da água. A partir dele os sistemas de planejamento são capazes de considerar as diferenças de atenuação de diferentes materiais, desde que seja inserida uma curva de densidade eletrônica (ED) por Unidade de Hounsfield (HU).

Para se obter a curva EDxHU é necessário que se disponha de um OS com diversas heterogeneidades. Estudos apontam variações dos valores de $\mathrm{HU}$ para diferentes tomógrafos e parâmetros de aquisição das imagens, como kV e filtros de reconstrução de imagem[2-6], o que deve ser levado em consideração ao se fazer a validação. 


\section{Materiais e Métodos}

O TRS430 recomenda alguns testes dosimétricos e anatômicos para o comissionamento dos sistemas para uso de correções de heterogeneidade $[3,7,8]$.

Após a inserção da tabela EDxHU, o primeiro teste a ser realizado é a verificação do contorno da superfície pelo sistema de planejamento. Esse teste não é dosimétrico, apenas garante que o contorno é o mais próximo possível do real.

Os casos dosimétricos são $[3,7,8]$ :

Caso 1 - Condição de referência com dados da $C T$ :

O propósito do teste é verificar um campo de referência $10 \times 10 \mathrm{~cm}^{2}$ com gantry e colimador a $0^{\circ}$.

As medidas são realizadas em várias profundidades ao longo do eixo central.

Caso 2 - Incidência oblíqua, falta de espalhamento e campos tangenciais:

O propósito deste teste é verificar o cálculo na ausência de condições de espalhamento pleno para campos tangenciais. O campo único tem o isocentro mais perto da superfície de forma que a radiação incidente tangencie a superfície do fantoma.

Caso 3 - Colimação significativa das bordas de campo:

Esse teste é realizado com um campo $14 \times 14 \mathrm{~cm}^{2}$ e colimador a $45^{\circ}$, bloqueado em um campo $10 \times 10 \mathrm{~cm}^{2}$, e tem como propósito verificar o cálculo para campo colimado.

Caso 4 - Quatro campos em box:

A técnica de 4 campos (Anterior, Posterior e 2 laterais) é utilizada em vários hospitais e o propósito do teste é verificar o cálculo para a dose entregue no isocentro pelos campos, separadamente e em conjunto.

Caso 5 - Expansão anatômica e colimação personalizada:

O teste visa verificar a colimação automática feita pelo sistema de planejamento. Um cilindro de $8 \mathrm{~cm}$ de diâmetro e $8 \mathrm{~cm}$ de comprimento deve ser criado centrado no ponto de cálculo, e a colimação do campo deve ser criada com uma margem de $1 \mathrm{~cm}$, resultando ao fim um campo $10 \times 10 \mathrm{~cm}^{2}$.

Caso 6 - Incidência oblíqua com campos irregulares e colimações no centro do campo:

O propósito é verificar o cálculo para campos irregulares com o centro bloqueado. O campo é colimado em forma de "L". São feitas medidas em campo aberto, no isocentro que fica na borda da colimação e abaixo da colimação.

Caso 7 - Três campos, dois com filtro e colimação assimétrica:

O teste visa verificar o cálculo com filtro e colimadores assimétricos. Os campos laterais possuem meio campo bloqueado e filtro. Os pontos de medida são em campo aberto e no isocentro.

Caso 8 - Campos não coplanares e teste da rotação da mesa e colimador:

O teste visa verificar o cálculo em situações em que o colimador ou a mesa estão angulados em diferentes ângulos de gantry. O campo anterior possui mesa angulada e os laterais são campos abertos apenas com angulação de colimador.

Todos os testes foram resumidos na tabela 1 . Alguns dos pontos de medida são dentro de heterogeneidades, o que dependendo do OS utilizado nem sempre é possível.

Tabela 1: Casos e campos de acordo com protocolo internacional $[3,7,8]$.

\begin{tabular}{|c|c|c|c|c|c|c|c|c|}
\hline Caso & \# Campos & \# Pontos & Setup & Tamanho do campo & Gantry & Colimador & Mesa & Modificadores \\
\hline 1 & 1 & 5 & SSD & $10 \times 10$ & 0 & 0 & 0 & Nenhum \\
\hline 2 & 1 & 1 & SAD & $10 \times 15$ & 0 & 0 & 0 & Filtro 45 \\
\hline 3 & 1 & 1 & SSD & $14 \times 14$ & 0 & 45 & 0 & Blocos ou MLC \\
\hline 4 & 4 & 3 & SAD & $10 \times 15 ; 8 \times 15$ & $0 ; 180 ; 90 ; 270$ & 0 & 0 & Nenhum \\
\hline 5 & 1 & 2 & SAD & $12 \times 19$ & 0 & 45 & 0 & Blocos ou MLC \\
\hline 6 & 1 & 3 & SAD & $10 \times 20$ & 45 & 90 & 0 & Blocos ou MLC \\
\hline 7 & 3 & 5 & SAD & $10 \times 12 ; 10 \times 6$ (assimétrico) & $0 ; 90 ; 270$ & 0 & 0 & Filtro $30^{\circ}$ \\
\hline 8 & 3 & 1 & SAD & $4 \times 16 ; 4 \times 4$ & $0 ; 90 ; 270$ & $0 ; 30 ; 330$ & $0 ; 270$ & Nenhum \\
\hline
\end{tabular}

O TRS430 [7] aponta que a validação pode ser realizada com um OS de fatias, o que tornaria impossível a realização de todos os testes recomendados, ou com um OS antropomórfico.

Diversos trabalhos [4, 6, 9-11] fazem a análise utilizando um OS de fatias. Bragga, C. eConwaya, J[12] fazem a análise em uma geometria complexa(OS antropomórfico) e em uma geometria simples(OS de fatias), em que são realizadas apenas medidas de pontos e perfil com feixe direto. Concluíram que tais medidas servem apenas para dar uma ideia da habilidade do algoritmo de predizer o efeito de heterogeneidades.

O serviço da UNICAMP conta com dois AL's, ClinaciX e Clinac 2100C, e com 4 tomográfos, sendo um deles um PET/CT.

Os tomógrafos utilizados passa por testes de controle de qualidade regularmente e possuem uma avaliação da resposta de HUxED.

Cinco OS's foram avaliados e estudados comparativamente para a construção do OS 
próprio: Modelo 002LFC (CIRS - Computerized Imaging Reference Systems), EasyBody (Euromechanics Medical $\mathrm{GmbH}$ ), Quasar (Modus Medical Devices Inc.), Fantoma 91235 (Standard Imaging Inc.) e PTW Inhomogeneity phantom (PTW Freiburg Inc.).

O OS modelo 002LFC da CIRS (Figura 1) possui dimensão de $43 \times 38 \times 23 \mathrm{~cm}^{3}$ e é constituído de material equivalente a tecido e implantes(inserts) equivalentes a osso, pulmão (ambos com cavidades para câmara de ionização) e água.

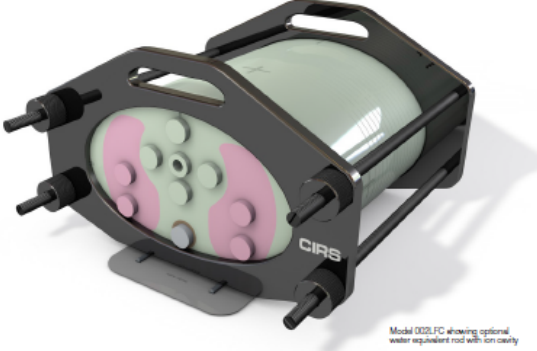

Figura 1: OS 002LFC da CIRS-Computadorized Imaging Reference Systems [13].

O OS EasyBody (Figura 2) foi desenvolvido pela Euromechanics Medical $\mathrm{GmbH}$, Alemanha. Consiste em um cubo que pode ser expandido lateralmente para fornecer uma forma de abdome. O material é composto de água sólida e possui dimensões de 36 × $18 \times 18 \mathrm{~cm}^{3}$. O OS possui diversas placas que podem ser rearranjadas. Possui implantes que acomodam câmaras de ionização para medidas em qualquer ponto. Filmes podem ser inseridos transversalmente entre as placas.

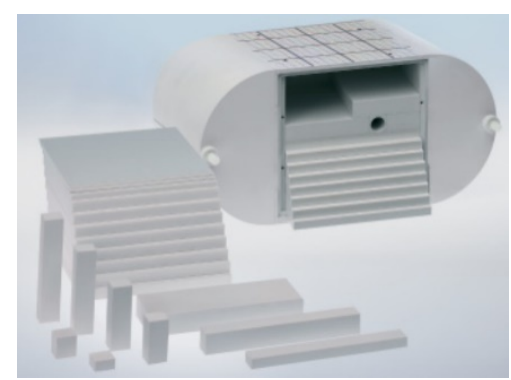

Figura 2: OS EasyBody da Euromechanics Medical GmbH[14].

O OS Quasar (Figura 3) é um produto da Modus Medical Deviceslnc, Canadá. É feito de acrílico de tamanho $30 \times 12 \times 20 \mathrm{~cm}^{3}$. Possui três aberturas cilíndricas de $8 \mathrm{~cm}$ de diâmetro para implantes de acrílico ou de baixa densidade (simulando pulmão). Possui seis aberturas menores, de $2 \mathrm{~cm}$ de diâmetro, para implantes de osso ou acrílico, com cavidade para inserção da câmara de ionização. O conjunto possui também um adaptador com cinco densidades eletrônicas diferentes para calibração da tomografia.

O OS 91230/91235 (Figura 4), da Standard Imaging é diferente dos demais e é feito de 6 placas de $3 \mathrm{~cm}$ de espessura, e de dimensão $30 \mathrm{x}$ $45 \mathrm{~cm}^{2}$. Pode ser adquirido em água virtual ou acrílico. Duas das placas possuem um material equivalente a pulmão. Em todas as 6 placas há um furo para inserção da câmara para realizar as medidas. Um implante de material equivalente a osso também pode ser inserido.

O OS da PTW, Freiburg Inc. (Figura 5), Alemanha, conta apenas com uma fatia que possui 3 implantes equivalentes a osso, pulmão e tecido. O tamanho da placa é de $30 \times 30 \mathrm{~cm}^{2}$ com $2,5 \mathrm{~cm}$ de espessura e as medidas podem ser realizadas com filmes ou com câmaras de ionização se se possuir placas de água sólida com cavidades para câmara.

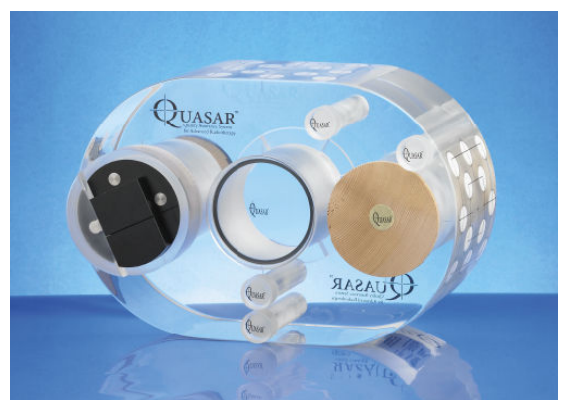

Figura 3: OS Quasar da Modus Medical Devices Inc. [15]

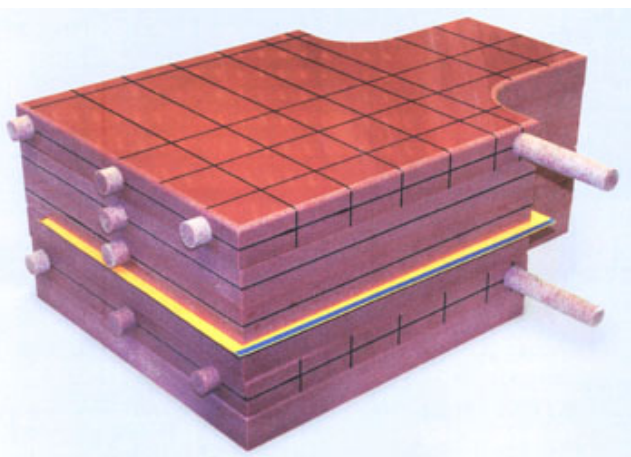

Figura 4: OS 91230 da Standard Imaging[16].

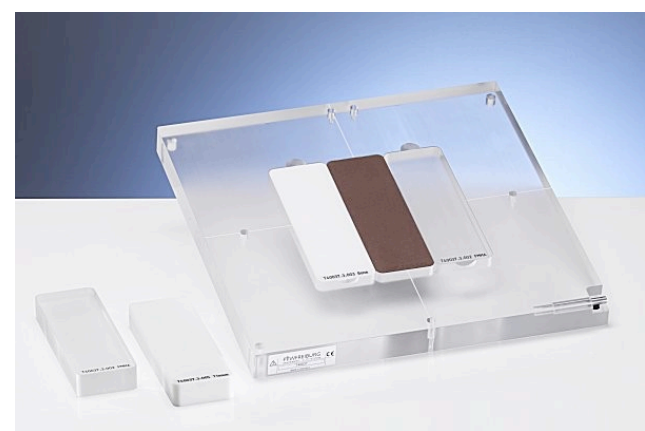

Figura 5: OS da PTW Freiburg Inc. [17]

A tabela 2 apresenta todos as características dos OS's estudados.

Algumas das características encontradas em OS's comerciais foram adotadas para a construção do OS, como por exemplo, as dimensões e os implantes.

Apesar de diversos estudos terem sido realizados com OS's de fatias [4, 6, 9-11], optou-se nesse estudo por um OS mais complexo, de forma que seja possível se medir campos laterais. 
Tabela 2: Fantomas comerciais analisados com informações que foram utilizadas no estudo

\begin{tabular}{|c|c|c|c|c|c|}
\hline Fabricante & CIRS Inc. & $\begin{array}{l}\text { Euromechanics } \\
\text { Medical GmbH }\end{array}$ & $\begin{array}{l}\text { Modus Medical } \\
\text { Devices Inc. }\end{array}$ & Standard Imaging Inc. & PTW Freiburg Inc. \\
\hline Fantoma & 002LFC & Easybody & Quasar & $91235 / 91230$ & LACO \\
\hline Dimensões & $30 \times 30 \times 20$ & $36 \times 18 \times 18$ & $30 \times 12 \times 20$ & $30 \times 45 \times 18$ & $30 \times 30 \times 2,5$ \\
\hline Material & $\begin{array}{l}\text { Água } \\
\text { sólida }\end{array}$ & Água sólida & Acrílico & Água virtual/acrílico & Acrílico \\
\hline $\begin{array}{l}\text { Número de inserts de } \\
\text { ED certificados }\end{array}$ & 5 & 4 & 5 & Nenhum & Nenhum \\
\hline $\begin{array}{c}\text { Permite incidências } \\
\text { oblíquas? }\end{array}$ & Sim & Sim & Sim & Sim & Não \\
\hline
\end{tabular}

O OS foi projetado no Google SketchUp e possui as seguintes placas:

- 6 placas de acrílico com $30 \times 30 \times 2 \mathrm{~cm}^{3}$, duas das quais com cavidade para câmara

- 9 placas de acrílico com $30 \times 5 \times 2 \mathrm{~cm}^{3}$, uma das quais com cavidade para câmara

- 2 placas de acrílico com $30 \times 12,5 \times 2 \mathrm{~cm}^{3}$

- 12 placas de cortiça com $30 \times 7,5 \times 1 \mathrm{~cm}^{3}$

- 1 placa de alumínio com $30 \times 5 \times 2 \mathrm{~cm}^{3}$

O processo de construção do OS ficou em responsabilidade do fornecedor contratado para fazer o serviço e os materiais, assim como o processo, podem variar dependendo as disponibilidades da fabricação.

A câmara utilizada para a validação foi uma Exradin A12, da Standard Imaging, e por isso a cavidade para câmara possui $1,3 \mathrm{~cm}$ de diâmetro e $17 \mathrm{~cm}$ de comprimento [18]. A câmara, com a capa de buildup, foi inserida até o fim da cavidade de forma a ficar no meio do OS.

As densidades eletrônicas do alumínio e da cortiça não foram medidas, mas são aproximadamente 0,2 e 2,3 [19-21] em relação a da água, respectivamente.

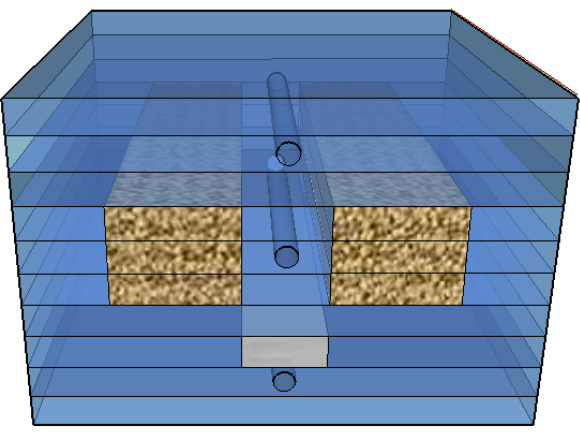

Figura 6: Projeção do OS criado no software, onde é possivel ver as diferentes heterogeneidades

Em cada caso foram feitas medidas nos pontos de interesse e calculado o percentual de variação da dose medida com a dose calculada pelo TPS.

\section{Resultados}

O OS construído custou cerca de $\mathrm{R} \$ 3.000,00$ enquanto um OS adquirido comercialmente custa em torno de U\$20.000,00.

O OS construído tem dimensões $30 \times 30 \times 16$ $\mathrm{cm}^{3}$ e é possível observar a comparação de um corte tomográfico deste com o OS comercial da Standard Imaging, figura 7.

Os pontos de medida estão apresentados na figura 7 por cruzes azuis. Cada fatia era substituída por uma com cavidade para câmara na hora das medições. As medidas nesse OS, assim como em alguns OS's comerciais, não são feitas dentro das heterogeneidades (ou mesmo em interfaces de heterogeneidades) e sempre após as mesmas, em meio de acrílico.

Todos os testes foram realizados para a validação do OS em dois aceleradores lineares. O sistema para planejamento utilizado foi o $\mathrm{XiO}$ versão 4.70 e algoritmo presente no sistema de planejamento que melhor calcula heterogeneidades é o Superposition $[8,22]$

Os resultados do percentual de variação da dose medida com a dose calculada pelo sistema de planejamento para todos os tomógrafos disponível na UNICAMP são mostrados nos gráficos das Figuras 8 e 9 para feixes de 6 e 10 MV, respectivamente. É possível observar que os pontos medidos no OS criado não são todos os pontos recomendados pelo TRS-430, tabela 1 . Tais pontos variam de acordo com o OS utilizado, que no caso conta com 4 posições para inserção da câmara de ionização.

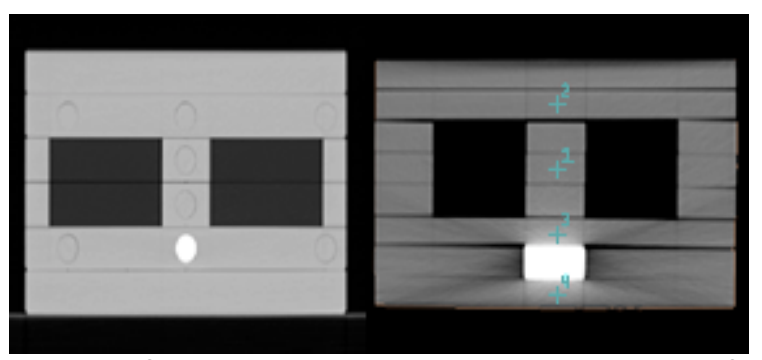

Figura 7: Comparação de corte axial da imagem tomográfica do OS91230 comercial da Standard Imaging (esquerda), com OS criado (direita), com as cruzes nos 4 pontos de medição utilizados na validação.

Os limites de aceitação estão de acordo com o estipulado no TRS-430.

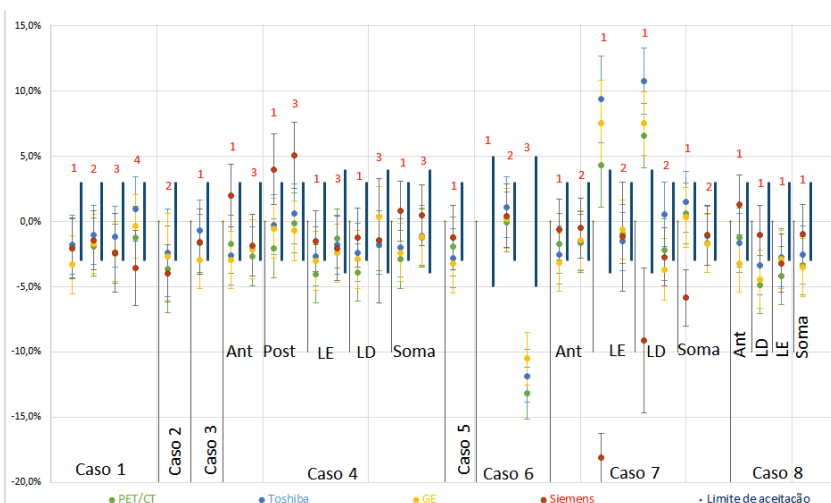

Figura 8: Resultados, para 6MV (ClinaciX), de todos os aparelhos de tomografia disponível no serviço, do percentual de variação da dose medida com a dose calculada pelo sistema de planejamento de todos os pontos de medidas (1-4), com barras dos limites de aceitação de cada teste. [7, 8] 


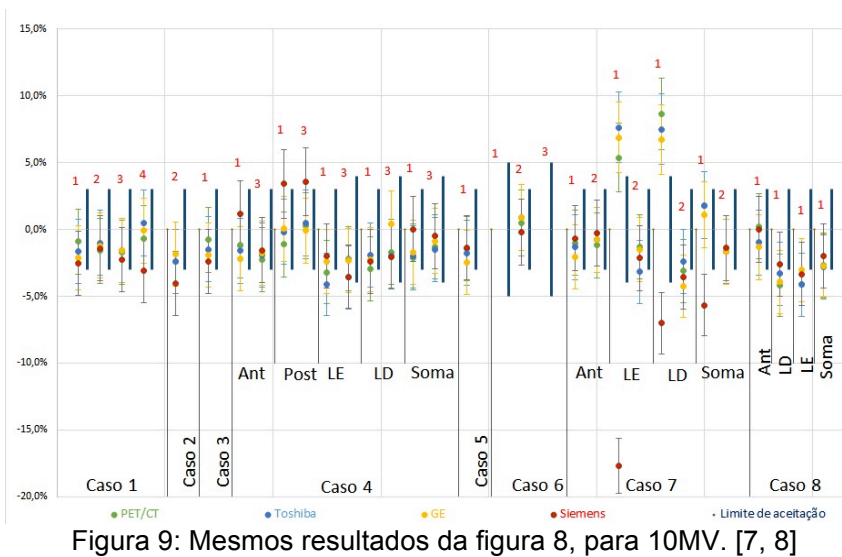

\section{Discussão}

As doses medidas pela câmara de ionização encontram-se dentro dos limites aceitáveis [8] em todos os pontos que estão em campo aberto, como pode ser visto nos gráficos das figuras 8 e 9 . Nos casos 6 e 7, que apresentam pontos de medidas na borda do campo, as diferenças entre as doses medidas e a dose calculada ficaram muito além do valor esperado, como é possível ver em ambos os gráficos. Isso se deve ao alto gradiente de dose que se faz presente nas bordas e à falta de precisão geométrica das medidas, mas é possível ver que a soma dos campos no caso 7 está dentro do limite, apesar do grande desvio nos campos laterais.

Gershkevitsha e colaboradores [8] realizaram os mesmos testes dosimétricos que os apresentados, para diferentes tipos de algoritmos. Pode-se ver que os erros podem variar significativamente com o tipo de algoritmo utilizado, mas em região de penumbra os desvios são maiores que na região de campo aberto, o que é esperado por ser uma região com alto gradiente.

\section{Conclusões}

É possível a construção de um OS a um custo abaixo do preço encontrado no mercado, de forma que possibilite a realização de todos os testes exigidos por protocolos internacionais, assim como futuros testes de controle de qualidade. A densidade eletrônica dos materiais utilizados não precisa ser conhecida para a validação da correção de heterogeneidade. É necessário, no entanto, um OS com diversas heterogeneidades cujas densidades eletrônicas sejam conhecidas para que seja possível a construção da curva de HUxED a ser inserida no Sistema de planejamento [5].

\section{Referências}

1. Engelsmana, M., et al. Impact of simple tissue inhomogeneity correction algorithms on conformal radiotherapy of lung tumours. Radiotherapy and Oncology, Vol. 60, pp. 299-309, 2001.

2. Guan, Huaiqun, Yin, Fang and Kim, Jae Ho. Accuracy of inhomogeneity correction in photon radiotherapy from CT scans with different settings. Phys. Med. Biol., Vol. 47, pp. N223-N231, 2002.

3. TECDOC 1583. Commissioning of Radiotherapy Treatment Planning Systems: Testing for Typical External Beam. Viena : IAEA Publications, 2008.
4. Zurl, B., et al. Hounsfield units variation: Impact on CTdensity based conversion tables and their effects on dose ditribution. Strahlenter Onkologie, Vol. 190, pp. 88-93, 2014.

5. Saw, Cheng B., et al. Determination Of Ct-To-Density Conversion Relationship For Image-Based Treatment Planning Systems. Medical Dosimetry, Vol. 30, No. 3, pp. 145-148, 2005.

6. Ragonezi, Aline, et al. Impacto Das Tabelas De Correção De Heterogeneidades Em Sistemas De Planejamento Radioterápico. In: Anais do XXI Congresso Brasileiro de Física Médica, Florianópolis : s.n., 2016.

7. IAEA, International Atomic Energy Agency.Technical Report Series 430. Commissioning And Quality Assurance Of Computerized Planning Systems For Radiation Treatment Of Cancer. Viena : IAEA Publications, 2004.

8. Gershkevitsha, E., et al. Dosimetric verification of radiotherapy treatment planning systems: Results of IAEA pilot study. Radiotherapy and Oncology 89, pp. 338-346, 2009.

9. Carrascoa, P., et al. Comparison of dose calculation algorithms in slab phantoms with cortical bone equivalent heterogeneities. Med. Phys. Vol. 34 No. 8, pp. 3323-3333, 2007.

10. Mackie, T., et al. Lung dose corrections for 6- and 15-MV $\mathrm{x}$ ray. Medical Physics, Vol. 12, pp. 327-332, 1985.

11. Santos, Gabriela Reis. Comparação de algoritmos computacionais de cálculo de dose em radioterapia aplicada aos tumores de pulmão. Tese de Doutorado. Universidade de São Paulo, Faculdade de Medicina: s.n., 2011.

12. Bragga, C. and Conwaya, J. Dosimetric verification of the anisotropic analytical algorithm for radiotherapy treatment planning. Radiotherapy and Oncology 81 , pp. 315-323, 2006

13. Data Sheet IMRT Torax Phantom Model 002LFC. [Online] Computerized Imaging Reference Systems, Inc., 2013. http://www.cirsinc.com/file/Products/002LFC/002LFC\%20D S\%20090116.pdf. [acessado em Julho 2016]

14. Technical Specificartions IMRT Phantom. [Online] IBA, 2009.http://www.ibadosimetry.com/sites/default/files/brochure/l mRT Phantom 01.pdf. [acessado em Julho 2016]

15. Ūser's Guide of Quasar Multi-purpose Body Phantom. Modus Medical Devices Inc., 2010.

16. Manual IMRT Dose Verification Phantom REF 91230/91235. [Online] Standard Imaging Inc., 2006. http://www.standardimaging.com/uploads/manuals/8033607_IMRT_Phantom.pdf.[acessado em Julho 2016]

17. Technical Specifications PTW Inhomogeneity Phantom. [Online] PTW Freiburg GmbH, 2012. http://www.lacoonline.com/LACO_products/PTWInhomogen eityPhantom.pdf. [acessado em Julho 2016]

18. Data Sheet EXRADIN A12. [Online] Standard Imaging, 29 de Março de 2011. http://atlas.physics.arizona.edu/ kjohns/teaching/phys586/s 11/pdf/ExradinA12Specs.pdf. [acessado em Julho 2016]

19. Kohno, R., et al. Dosimetric verification in inhomogeneous phantom geometries for the $\mathrm{XiO}$ radiotherapy treatment planning system with 6-MV photon beams. Radiol Phys Technol, pp. 2:87-89, 2009.

20. Vassiliev, Oleg $\mathrm{N}$, et al. Validation of a new grid-based Boltzmann equation solver for dose calculation in radiotherapy with photon beams. Phys. Med. Biol., Vol. 55, pp. 581-598, 2010

21. Wong, J. and Purdy, J. On methods of inhomogeneity corrections for photon transport. Medical Physics, Vol. 17, pp. 807-814, 1990

22. Animesh. Advantages of multiple algorithm support in treatment planning system for external beam dose calculations. Journal of Cancer Research and Therapeutics, Vol. 1, No. 1, January-March, 2005, pp. 1220, DOI: $10.4103 / 0973-1482.16085$.

Contato:

Thallis Alves Santos

e-mail: thallis.alves@yahoo.com.br 